\title{
Lineage Explanations: Explaining How Biological Mechanisms Change Brett Calcott
}

\begin{abstract}
This paper describes a pattern of explanation prevalent in the biological sciences that I call a 'lineage explanation'. The aim of these explanations is to make plausible certain trajectories of change through phenotypic space. They do this by laying out a series of stages, where each stage shows how some mechanism worked, and the differences between each adjacent stage demonstrates how one mechanism, through minor modifications, could be changed into another. These explanations are important, for though it is widely accepted that there is an 'incremental constraint' on evolutionary change, in an important class of cases it is difficult to see how to satisfy this constraint. I show that lineage explanations answer important questions about evolutionary change, but do so by demonstrating differences between individuals rather than invoking population processes, such as natural selection.
\end{abstract}

\section{Introduction}

2 Turning a 'Scale' into a 'Plume'

3 Lineage Explanations in Biology

3.1 The evolution of eyes

3.2 The evolution of feathers

4 The Two Dimensions of a Lineage Explanation

4.1 The production dimension

4.2 The continuity dimension

4.3 The dual role of the parts

5 Constraining the Explanations

6 Operational and Generative Lineages

7 Explaining Change Without Populations

8 Conclusion

(c) The Author (2008). Published by Oxford University Press on behalf of British Society for the Philosophy of Science. All rights reserved. doi:10.1093/bjps/axn047 For Permissions, please email: journals.permissions@oxfordjournals.org Advance Access published on December 10, 2008 


\section{Introduction}

This paper describes a pattern of explanation prevalent in the biological sciences that I call a 'lineage explanation'. These explanations often appear as a diagram resembling a cartoon strip. Each stage shows how some mechanism worked, and the differences between each adjacent stage demonstrates how one working mechanism, through minor modifications, could be changed into another working mechanism. This series of mechanisms, as a whole, shows the plausibility of certain trajectories through phenotypic space. Demonstrating this plausibility is an important goal, for it is widely accepted within evolutionary biology that there is an 'incremental constraint' on evolutionary explanations of change. In an important class of cases, it is difficult to see how to satisfy this constraint. A distinctive virtue of lineage explanations is that they show how change can be incremental, even in these difficult cases.

Lineage explanations are an answer to a distinctive kind of question about evolutionary change. The question 'how did biological mechanism $X$ change over time' is ambiguous. ${ }^{1}$ We could be asking about the process that drove change - a question to which natural selection, in part, provides an answer, or we could be asking a question about what particular modifications are responsible for the differences between organisms related by descent. Here, the process of natural selection has little to say. Instead, the explanation lies in the details of how the particular mechanism of interest worked, and how it changed over time. It is this second question that lineage explanations address.

Lineage explanations are particularly important in evolutionary developmental biology (evo-devo), where they are used to explain the origins of evolutionary novelty. The distinctive nature of explanation in evo-devo has been the subject of some philosophical attention (Wagner [2000]; Amundson [2001]; Winther [2005]). The account that I give here divides the landscape up in a unique way. For I take the primary distinction to be one between populationlevel and individual-level explanations, rather than between adaptationist and developmental explanations. As I shall show, non-developmental, adaptationist explanations can also use this pattern of explanation, particularly when they attempt to explain the origins of complex adaptations.

My approach in this paper will be to concentrate on outlining the general structure of these explanations. I provide some detailed examples, demonstrating the core aspects of these explanations and some important variations on how they can be constructed. These explanations are complex, so exploring their structure shall take much of the paper. I shall have little space to examine in detail how these explanations relate to more general issues of explanation,

1 See Amundson ([2001], [2005]) for some excellent discussion about the differing types of contrastive questions one might ask in biology. 
but I shall make some important connections in the final section. A deeper exploration of the issues shall have to wait.

I begin by outlining the core features of these explanations via a toy example in Section 2. In Section 3, I introduce two biological examples of lineage explanations. Section 4 explores the similarities between these examples, showing that they both possess the same core structure outlined in the toy example. Section 5 examines a number of different ways that these explanations might be constrained. In Section 6, I identify two variants of lineage explanations. I then look more closely at the type of explanation I have described in Section 7 , and finish with a brief summary of these explanations.

\section{Turning a 'Scale' into a 'Plume'}

This section is all about a particular type of puzzle. There is nothing biological or evolutionary about this puzzle. I use it to show the core structural features of a lineage explanation first, without being distracted by the 'gory details' of biology.

Here is one version of this type of puzzle. Given two words, the challenge is to transform one word into another by changing only a single letter at a time. Each of the intervening stages must also be a word. A valid word is any English word that isn't a name or an abbreviation. Here, for example, is one way to get from the word 'scale' to the word 'plume'.

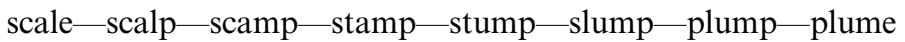

Beginning with 'scale', we change the last letter to 'p', forming 'scalp'. We then change the fourth letter in 'scalp' to ' $m$ ', forming 'scamp'. We proceed in the same manner, changing a single letter at a time, finally forming 'plume'. ${ }^{2}$

This is a very simple game, but it has a number of features I want to spell out. A solution to the puzzle describes a trajectory between two chosen words. This solution provided must meet two requirements. First, only a single letter may be changed at a time, so each adjacent word must share a significant number of letters in common. No big jumps are allowed. I'll call this a continuity requirement. Second, each intermediate stage must form an actual word. We cannot, for example, go from 'scale' to 'slale'. For even though this change meets the continuity constraint (a single letter change), 'slale' is not a valid English word. I'll call this a production requirement. At each stage in the trajectory the letters must produce some output that meets a particular criterion; in this case, the letters must form a valid English word.

2 For puzzle pedants: As far as I can work out, there is no trajectory between 'scale' and 'plume' with fewer stages than the example shown here, though there are other routes where the 'e' remains unchanged for the entire route. 
Fulfilling both of these requirements is achieved by manipulating the letters. The chosen letters and their organisation are what produce the word, and the difference between stages is brought about by changes in those same letters. We can say that the letters are simultaneously the components that produce some required output at each stage, and they are the grain of change that brings about the differences between each stage. This dual role played by the components due to the two requirements is a central feature of the puzzle.

Note that we could modify the puzzle in various ways by constraining how these requirements are fulfilled. Rather than using the entire English lexicon, I could limit the set of valid words in some manner: only words that appear in Shakespeare's 'Hamlet', for example. It is also possible to apply additional constraints between the stages (in addition to continuity). Perhaps a valid word must not only be drawn from a defined lexicon, but it must also sort alphabetically after the word in the previous stage (in this case, getting from scale to plume would not be possible, scale/scalp/scamp is okay). This additional sorting constraint between the stages in is terms of the words produced at each stage. But we could also constrain the way letter changes are made in a more restrictive manner: the first change must be on the first letter, the second change on the second letter, and so on. Or we might have a more interesting idea of what constitutes a continuous change; maybe treating letter exchanges within the word as continuous too (an organisational change, rather than a replacement). Many of these kinds of changes will make the puzzle harder (maybe even impossible), for they reduce or constrain the number of possible trajectories that meet the requirements. Nevertheless, the core of the puzzle remains the same: constructing a continuous trajectory of change in a set of components that produce some output.

One last puzzle variant will emphasize an important point. Imagine a puzzle where, instead of letters, there are numerals and mathematical symbols. The components make an equation (' $6 \times 4+1$ ', for example) and this equation produces a result (' 25 ', in this case). As with the letters, there will be constraints on what combination of symbols produce a valid equation. Now let us add an additional constraint on the relation between the stages: they must all produce the same result. So, we begin with two equations that both produce the result '9', and now we must find a series of one-step symbol changes between these equations where each equation in the series likewise produces the result ' 9 '. ${ }^{3}$

Here is the important point that this variant highlights: it is not simply changes in the output, but changes in how the output is produced that is central to the puzzle. For even if the series of outputs remains exactly the same, as it does in this previous example, there is clearly still a puzzle to solve. The question

3 No, I don't have an example of this. I suspect you might need a more liberal continuity rule to actually get some workable examples. 


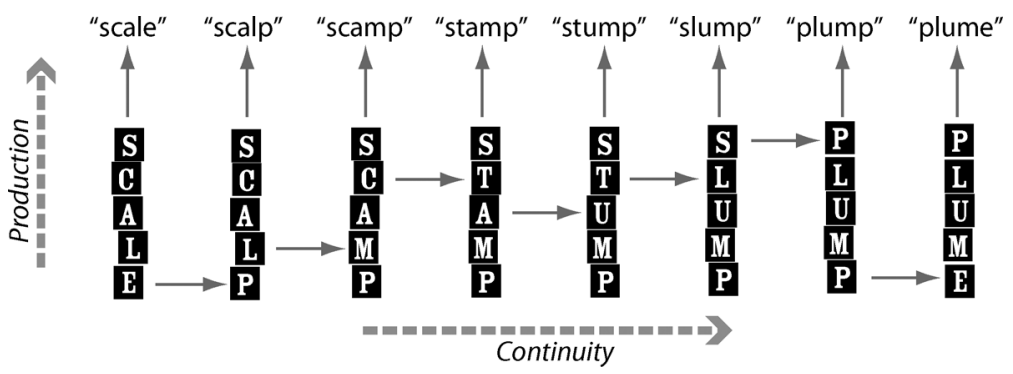

Figure 1. The two dimensions of a lineage explanation. Production runs vertically: each choice of letters and their organisation must make a word. Continuity runs horizontally: these same words change through a single replacement of the letters.

of change in how output is produced is what is puzzling, despite variations in the demands that can be made on the series of outputs. Simple membership of a group at each stage might be sufficient, or directional demands between stages may be imposed (the sorting constraint given above), or the outputs may have to stay the same (as in the equation example). These different demands on the output constrain the puzzle in important ways. But the puzzle itself lies in showing how production changes in a continuous manner.

These two requirements - production and continuity - form what I call the two dimensions of a lineage explanation (see Figure 1). The first dimension runs vertically through each stage of the trajectory, showing how the component letters meet the production requirement. The other dimension runs perpendicular, connecting each combination of letters via the continuity requirement. These two dimensions are what gives the letters this dual role as both the units of change and the components for constructing valid words.

I'll summarise the structure of this kind of puzzle in the following way. We begin by choosing two endpoints, and then show a series of stages that demonstrates how one could be changed into another. Each stage in the reconstruction must itself produce some output in virtue of the components that make it up, and each of the adjacent stages is related to one another by small continuous changes in those components. The dual requirements of puzzle mean that the components making up the puzzle must play two roles: both producing a result and providing the grain of change. These requirements may be constrained in different ways, by specifying particular conditions that must hold both within and between stages. Additional constraints can limit the number of possible trajectories in various ways. I'll return in more detail to both the requirements and the constraints on lineage explanations after presenting some biological examples.

It is also important to note what is not part of the solution to the word puzzle. The puzzle does not specify any particular process for producing the 
trajectory - how you go about finding a possible route from one word to another is up to you. You can start at one end and work your way to the other, or you could start at both ends and try and find some overlapping middle point. These details do not figure in the solution. It is also quite likely that, in the pursuit of a trajectory you will explore many dead-ends - partial trajectories to words where you can go no further. But these discarded paths do not figure in the solution to the puzzle either. What matters is simply that you show a trajectory that satisfies the two requirements of continuity and production. Any trajectory containing intervening stages that meets these requirements is sufficient.

We shall see that what is excluded from these solutions also has an analogue with the biological versions of lineage explanations. The population processes essential to driving the changes in lineages are excluded from the lineage explanations. This is not to say that they don't occur, or are not important. But lineage explanations focus on a different, and difficult, question. What matters in these explanations is demonstrating how, given certain constraints, a particular trajectory of change is plausible. What processes might be responsible for making these changes are omitted from the explanation.

\section{Lineage Explanations in Biology}

Biologists are often faced with puzzles resembling those in the previous section. They identify two organisms related by descent, yet these organisms differ in some aspect of behaviour, in the presence or absence of some feature, or perhaps in the different way that some common feature is produced. The puzzle is to figure out how something that works like this could have turned into something that works like that.

The explanations given for these kinds of problems consist of two dimensions. These two dimensions are (unsurprisingly) somewhat more complex than the puzzles in the last section. Rather than the production dimension consisting of some simple composition where letters form words, the production at each stage is performed by some kind of mechanism. I shall return in more detail to the issue of mechanistic explanation in Section 4.1. For now, however, it will be sufficient to say that a mechanism consists of a set of components (individual parts, processes, and their organisation) that produces some regular phenomenon (a behaviour, or a morphological form, for example). ${ }^{4}$ It is in virtue of gradual changes to these components that we make plausible a continuous trajectory of change in a lineage of organisms.

4 To avoid wordiness I will use the word 'components' to capture all the relevant details that are meant to make up a mechanism, such as entities, activities, and their organisation. Exactly what this list includes is still somewhat controversial (see Tabery [2004] for some discussion of this issue). 
Like the puzzle, there are some variants on how we might constrain the trajectory between the two endpoints. The output in each stage might be required to resemble a member of some series of fossils, or some related extant species. Directionality may be imposed on the series by appealing to the adaptive value of the phenomenon that mechanism produces. Or we might try to explain how the mechanisms underlying the development of an animal have changed over time whilst the adult form has remained largely the same - an example resembling the puzzle with the equation in the previous section. In each case, however, the puzzle to be solved is how gradual change could have occurred in the way a mechanism produces some phenomenon.

In what follows, I give two detailed examples of lineage explanations. The first is Nilsson and Pelger's account of eye evolution (Nilsson and Pelger [1994]). The second is Prum's account of feather evolution (Prum [1999]). ${ }^{5}$

\subsection{The evolution of eyes}

Nilsson and Pelger construct a model describing a possible route from a simple light-sensitive patch to a focussed lens eye (Nilsson and Pelger [1994]). They base their reconstruction on an idealised morphological model that is general enough to capture the structural details present in the eyes of many different organisms. Each successive stage shows some simple change from the previous one, and in each successive stage the eye is capable of producing better and better images. This improvement plays an important part of the model as it provides an adaptive constraint on the series. Nilsson and Pelger provide an estimate of the time that these transformations would take, based on the relative size of these small changes in morphology (shape, size, etc.). They conclude that, even with pessimistic parameters, such an eye could evolve in a few hundred thousand years.

The measure of an eye's adaptive value in this sequence is based on the spatial resolution that each morphological variant can produce. This is simply the ability for it to provide a sharp picture. According to Nilsson and Pelger, no matter what varied visual functions an eye might have in different organisms - such as movement tracking or pattern detection - spatial resolution is central to how well that eye can perform.

Each stage in Nilsson and Pelger's model has the same parts: a light-sensitive patch of cells sandwiched between a transparent layer and a dark pigmented layer. The parts undergo various modifications throughout the series from a

5 For some other clear examples of the two dimensions of lineage explanations, see the diagrams in the following: (Truman and Riddiford [1999]; Peel and Akam [2003]; Suzuki and Nijhout [2006]). For an example where the output of the mechanism stays the same, but the mechanism changes over time, see the discussion in Raff's chapter 'Building Similar Animals in Different Ways' (Raff [1996]). 


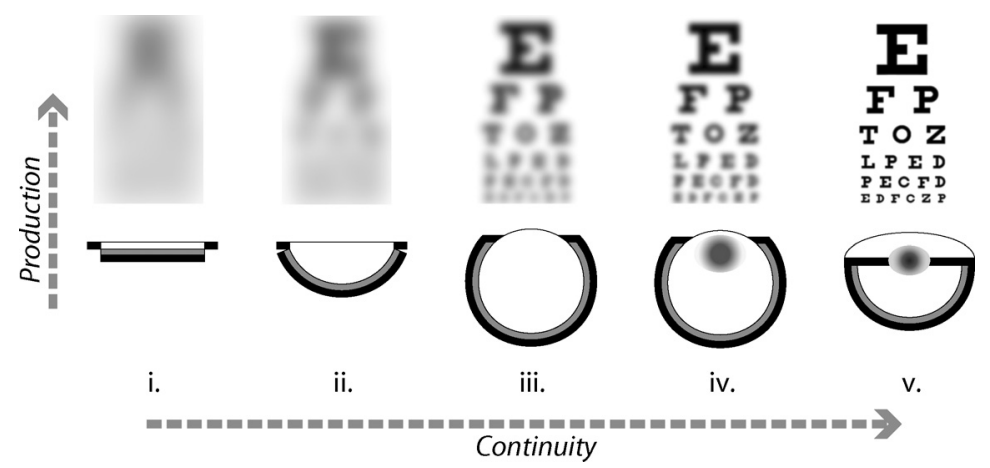

Figure 2. Five stages in eye evolution. Each small change increases the ability of the eye to produce a better image (as the 'eye chart' shows). As in the word puzzle, the two dimensions of the explanation are present. The parts, processes and their organisation in the eye explain this ability, and the small changes in the organisation of the components provide the continuity. Redrawn from (Nilsson and Pelger [1994]).

simple light-sensitive spot to a camera-lens eye. The stages proceed as follows (see Figure 2). ${ }^{6}$

(i) The three layers of cells begin as a flat light-sensitive spot.

(ii) A depression forms in the centre of the light-sensitive and pigmented layer (together, forming the retina), and the transparent layer deepens to fill this depression.

(iii) The opening towards the light then narrows. The eye now resembles a simple pin-hole camera.

(iv) An increase in the refractive index in a portion of the transparent layer produces a graded index lens that begins to focus the incoming light. ${ }^{7}$

(v) The light aperture narrows further to form an iris, and moves to the centre of curvature to produce a tightly focussed image on the retina.

The increase in spatial resolution over the first three stages relies on the narrowing of the aperture that permits light through and the increased curvature of the receptive surface. But the continued narrowing of the light aperture also lessens the total light that is received, producing a noisy image. At some point a trade-off is reached, where narrowing the aperture decreases the field of view

6 These stages are simplified from the original paper, but maintain the central changes. Nilsson and Pelger's diagram contained eight stages. I have compressed this to five stages that capture the key events.

7 Glass lenses have a constant refractive index. The transparent part of vertebrate eyes has a variable refractive index producing a graded index lens. 
for the receptors, but simultaneously increases the noisiness of the image. At this stage a lens is required to increase resolution which appears in stage (iv). Before this stage, the refractive index of the transparent layer of cells has been homogeneous. The remaining stages produce a graded index lens, and the shape of the receptive surface takes on a parabolic shape.

Nilsson and Pelger show that these modifications can produce a roughly linear increase in spatial resolution. So there is no place where a large morphological change is required to produce a small adaptive payoff. Nilsson and Pelger emphasise the importance of small changes providing a smooth gradient on which selection can act.

This reconstruction seems plausible as only a small change is needed in the morphology of the eye in each stage. But there is no guarantee that small changes in morphospace can be generated by small changes in the genotype. It could be the case that what appears as a small difference in morphology required some large change or reorganisation at the genotypic level. So this explanation makes an implicit bet: that there is genotypic variability available that maps smoothly to these small morphological changes.

These assumptions are at least partially supported by providing other evidence. Eyes resembling each of these stages can also be found in living organisms. Moreover, a complete series of these eyes can be found within a single phylum (both the annelids and the molluscs). So there is at least some prima facie evidence that the requisite variability is available. The origin of the lens is also made more credible as the change in refractive index is caused by proteins that have other cellular functions. So they may have been co-opted rather than have arisen de novo.

\subsection{The evolution of feathers}

The complex structure of feathers is regarded as an example of a morphological novelty (Prum [1999]; Müller and Wagner [2003]). In what follows, I summarise an account of the evolution of feathers from (Prum [1999]). Prum takes a purely developmental approach to explain the evolution of feathers, explicitly ignoring their adaptive function.

Feathers are a complex branched epidermal growth. The main branch is called the rachis, off this come the barbs, consisting of a barb ramus and variably shaped projections called barbules (see Figure 3A). Although feathers consist of a branched structure, they do not grow by branching at the tips in the way that trees do. Instead, they grow from the base, like hair or fingernails, and the different levels of branching are each constructed by various mechanisms located in the feather follicle. This follicle is a cylindrical invagination in the epidermis - like the indentation you would get on your skin if you held a small metal pipe on it for a while. Feather growth is fed from nutrients in the follicular 


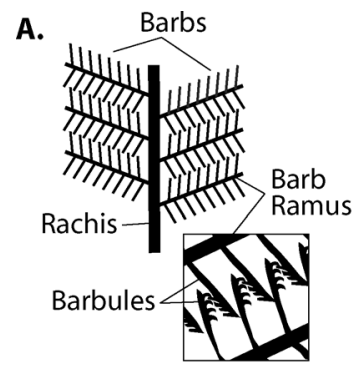

B.

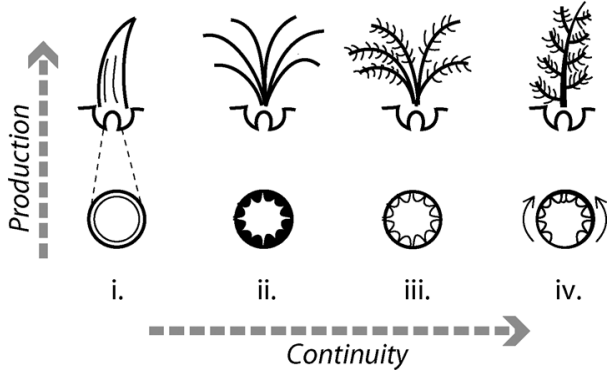

Figure 3. Feather structure and evolution. (A) The hierarchical structure of a typical feather. The closeup shows the finer detail of the interlocking barbules that form the planar vane for a flight feather. (B) Proposed evolution of the feather. On the bottom are cross sections of the follicle collar during feather development. The changes here explain the resultant changes in feather form on the top row. Again, the two dimensions of the explanation are present. Redrawn from (Prum [1999]) and (Yu et al. [2002]).

cavity (the base of the indentation), the form is constructed on the follicle collar (the outside of the tube projecting in the middle), and is displaced upward to produce a keratin matrix that constitutes the mature feather. The production of the complex branched structure is derived from the interaction of several processes of cellular differentiation occurring on the follicle collar.

Prum proposes a series of stages to explain the evolution of feathers based on changes that occurred in the feather follicle. ${ }^{8}$ Each of these changes in the feather follicle produces a new stage in feather morphology. So although the target of this explanation is the changing form of feathers, the explanation is given in terms of the follicle.

A simple analogy can help to understand the relevance of these modifications. We can think of the feather as being extruded from the follicle, rather like toothpaste coming out of a tube when you squeeze it. If someone asks how stripy toothpaste is produced (a phenomenon causing much puzzlement when I was a child), we tell them about the modifications to the nozzle of the toothpaste tube that lay down the stripes as the toothpaste is squeezed out. The modifications that Prum proposes can be likened (very roughly) to modifications to the nozzle of the toothpaste tube. They are changes in the components of the mechanism that generate the form of the feather (see Figure 3B).

8 See (Prum [1999]) for details. Here, I have reproduced the stages that Prum labelled I through III, and assumed that IIIb comes before IIIa. This is the same sequence suggested as more likely by Yu et al. ([2002]). 
(i) The ancestral follicle consists of a simple cylindrical indentation. From this, the initial growth produces a hollow cylinder. (Imagine a toothpaste nozzle which has a round piece stuck in the middle).

(ii) The epidermal collar then differentiates into longitudinal barb ridges. This produces a tuft of unbranched barbs (like stripy toothpaste, but only the stripes come out).

(iii) Peripheral paired barbule plates then appeared on the sides of the barb ridges. These produced the first fine-level structure on the outgrowths, the rami and the barbules (the toothpaste analogy runs out of steam here).

(iv) The point of barb growth becomes displaced as the barb is growing - producing helical growth around the follicle (following the arrows in the Figure 3B (iv)). New growth begins at the barb locus and eventually comes together to fuse and produce the rachis. This change created the large-scale branch structure, and enabled an indeterminate number of barbs to be produced for any outgrowth.

Prum's reconstruction seems plausible because, despite feather form changing radically, there are only small changes needed in the follicle to produce these changes. In addition, as with the eye, there are examples of these kinds of intermediate structures being produced in extant organisms. For example, the downy feathers found on birds resemble stage (iii).

We might think that the inclusion of developmental information in the feather example means that it somehow 'goes deeper' than the adaptive account of the eye example. But although Prum's account includes some developmental information, it still makes the same kind of bets that the eye account does. What produces change in feather morphology is small changes in the follicle that produce these feathers. But no account is given of how these small changes in the follicle map onto genotypic changes. So Prum is still making the same kind of bet about the genotype to phenotype mapping as Nilsson and Pelger. As with the eye example, Prum supports his assumptions by providing comparative information. Each of the hypothesised feathers can be found in extant birds, as well as the developmental mechanisms responsible for the production of the variants in feather shape.

\section{The Two Dimensions of a Lineage Explanation}

Nilsson and Pelger's explanation of eye evolution and Prum's explanation of feather evolution are both examples of lineage explanations. In this section, I look more closely at the resemblance these examples have to the puzzles in the first section, and also the resemblance they have to each other. I'll do this by showing how, like the puzzles in Section 2, there are two dimensions present 
in these explanations, and the components making up the explanations play a role in both of these dimensions.

\subsection{The production dimension}

The production requirement in both of these examples is fulfilled by showing how some mechanism produces some phenomenon. The phenomenon produced in each stage in Nilsson and Pelger's series of eyes was a focussed image, with some degree of spatial resolution. The phenomenon produced by each stage in Prum's series of feathers was some variant of feather structure. Like the word puzzle, this output or phenomenon was produced by the components present at that particular stage: the combination and configuration of cell layers in the eye, or the construction and processes occurring in the feather follicle.

Describing how each stage produces these phenomena results in a series of self-contained explanations. The last stage of the eye, for example, explains the spatial resolution of that particular type of eye by providing facts such as the ellipsoid shape of the layers of cells, the amount of light that this lets in and the optical density of the transparent cells. A similar independent explanation can be given for the other stages in the eye example. If we found some creature with an eye resembling, say, Figure 2 (ii), then the details that Nilsson and Pelger provide about that stage would explain how that particular creature had the visual acuity it did. Similarly, if we found a creature with feathers resembling those in Figure 3 (iii), the developmental explanation offered by Prum about that stage would explain why those feathers had the form that they did. So a lineage explanation contains a series of smaller, independent, explanations.

These independent explanations at each stage are mechanistic explanations. There are a number of closely related modern accounts of mechanistic explanation (Glennan [1996]; Machamer et al. [2000]; Bechtel and Abrahamsen [2005]). The goal of these accounts is to capture the practices of many of the life sciences, where to give an explanation is just to give a description of a mechanism (Machamer et al. [2000]). Here is a definition of a mechanism from (Bechtel and Abrahamsen [2005]):

\footnotetext{
A mechanism is a structure performing a function in virtue of its component parts, component operations, and their organization. The orchestrated functioning of the mechanism is responsible for one or more phenomena (Bechtel and Abrahamsen [2005]).
}

The individual stages in both Nilsson and Pelger's account of eye evolution and Prum's account of feather evolution fit well with mechanistic explanation. In each stage of the model of eye evolution, the component parts (the different cell types) and their relations and operations (the effect their form has on light) 
explain how that eye can achieve the visual acuity it does. In each stage of the model of feather evolution, the component parts (of the follicle) and their relations and operations (differential cell death, helical growth) explain the form of the feather that is produced. It is not surprising that these intermediate stages fit well with mechanistic explanation. The goal of the mechanistic account of explanation was to deal with exactly the kinds of scientific domain present here: developmental biology and physiology.

One feature of mechanistic explanation emphasised by some authors is their hierarchical nature. A component within one mechanism may itself be a mechanism, and hence be the subject of another explanation. Given a particular mechanism, we can choose to decompose it in different ways by choosing different levels of description (Bechtel and Richardson [1993]).

What guides the scientist in choosing the 'right' level of description to explain the phenomena of interest? There appear to be four ways of choosing the 'right' level that are described in the literature. First, the nested descriptions of mechanisms may simply 'bottom out' at some level relevant to the scientific domain of interest (Machamer et al. [2000]). Second, depending on the particular mechanism being described, the 'working entities' playing a role in the mechanism may pick out different levels of descriptions. Thus, in the mechanism of meiosis, it is the chromosomes that are the working entities, whereas in other molecular processes (such as transcription) the working entities will be pieces of DNA that (partially) constitute the chromosome (Darden [2005]). Third, scientists may provide incomplete descriptions by abstracting away certain details. The purpose of this may be to give a more compact description, or it may be that they wish a more general description that unifies a set of particular mechanisms (Machamer et al. [2000]). Finally, the process of decomposing a mechanism to provide an explanation is an ongoing one (Darden [2002]; Bechtel and Abrahamsen [2005]). So we might 'black-box' a particular component of a mechanism that we don't yet fully understand, though we can describe enough of its apparent behaviour for it to play a role in some higher level description.

As can be seen, the choice of a particular decomposition depends on different things. In the first case, we are guided by our broad domain of interest. In the second case, it is a narrower domain of interest, that of a particular mechanism, that guides us. In the third case, it is the role that we wish our explanation to play. In the last case, we are constrained by the current limits to our knowledge.

This ability to decompose a mechanism in different ways plays an important part in how these mechanisms contribute to a lineage explanation. We'll see in the next section that the way we choose to decompose the mechanism in a lineage explanation is guided by additional concerns not mentioned above, concerns about relations of change between mechanisms. 


\subsection{The continuity dimension}

A lineage explanation fills in plausible intervening stages between two phenotypic endpoints that at first glance may look very different. We make a large change credible by positing a set of intermediate stages. But this is only part of the story. A mechanism is decomposed into a number of components, so we can explain this continuity in terms of the components, rather than at the focal level of the trait. Continuity between stages is achieved because the components used to describe a mechanism can be credibly redeployed or reorganised to construct the mechanism at a successive stage. So the vertical dimension-how we decompose the mechanisms into components - will be tied to the horizontal dimension - the ability of those components to provide a continuous story between each stage.

For example, in the model of eye evolution, the same three layers of cells are present throughout the stages. Their shape, size and relation to one another are modified, but the credibility of continuity between the stages is justified because there is no radical change in parts, or their properties, during the transitions. The decomposition of the eye into these simple layers of cells not only explains the visual acuity of the eye, but it is also the right level at which we can explain the small differences that enabled increased acuity in the next stage. Similarly, the parts and organisation of the feather follicle - such as the barbules ridges and the helical growth - simultaneously explain the production of a particular feather form and provide a set of parts by which to account for the changes between different feather forms.

Decomposition supplies an essential ingredient to explaining continuity between stages, over and above adding more intermediate stages. A straightforward gradualist proposal might be to explain change by simple operations on the entire focal trait, such as the Cartesian scaling and deformation famously portrayed in D'Arcy Thompson's 'On Growth and Form' (Thompson [1961]). But by decomposing the trait into some mechanistic description, we can then explain change in terms of the components at this lower level. Now, deformation of the individual components can be employed to explain change. More importantly, we can further explain change in terms of the addition, deletion, duplication, reuse and reorganization of components - something not possible if we remain at the singular focal level.

For example, note that the transparent layer of cells in the eye example, originally proposed to serve as a protective layer, gains the additional function of focussing light. So, although the function of the eye remains constant throughout all the stages, the components that make up the eye have changed their function. A modification to a single part of the eye plays a role in changing the function of the entire mechanism. Without decomposing the mechanism into parts it would not be possible to show this kind of change. 


\subsection{The dual role of the parts}

Letters played a dual role in the puzzles in Section 2, participating in both dimensions of the puzzle. The letters chosen, and how they were organised produced the word at each stage, and small changes in the letters enabled a continuous change between stages. This dual role is similarly present in lineage explanations in biology. The components making up the mechanisms produce the relevant phenomena at each stage, and they serve as the grain of change for enabling continuity between stages.

This dual role of the components in a mechanism distinguishes lineage explanations from other types of biological explanation. It also provides the basis for the recent focus on a number of concepts such as modularity and robustness in such fields as evo-devo. I'll show this by drawing some analogies between explanation in biology and planning in engineering.

Consider some typical questions in proximate biology: how geckos stick to the ceiling (Autumn and Peattie [2002]), how ants find their way home (Wehner [2003]) or how fleas jump (Rothschild et al. [1975]). Providing an explanation for phenomena such as these goes hand-in-hand with knowing how we could, at least in principle, build something that does a similar job in a similar way. So if we really understand how a gecko sticks to the ceiling, we can build something that sticks to the ceiling like a gecko. ${ }^{9}$ Furthermore, the description of such mechanisms, often in the form of a diagram, provides (at least the outline) of a plan for how to build a machine that could reproduce these abilities. The suggestion (surely not original) is that there is some connection between explaining how a mechanism does $X$, and knowing how to build a mechanism that does $X$ in a similar way. Perhaps this connection is not perfect, but it will suffice to make clear the importance of adding a continuity requirement to both explanations in biology and design in engineering.

Note that the kinds of biological questions above are only about a production dimension: describing how a mechanism produces some output, or does some job. Suppose we introduce something like a continuity dimension to the picture. What would this mean for an engineering task? As it happens, the addition of a continuity dimension is exactly what occurs in modern software engineering practice. The challenge is not simply to build some software that performs a particular task - the software must be built in such a way that it is easily modifiable. This is important as the functionality required from the software can change, sometimes radically, even whilst the software is still undergoing

9 In this case, this is exactly what has been done: gecko-like sticky material has been made based on the same principles as gecko feet. 
development. ${ }^{10}$ So the engineering task of software development often presents a dual challenge: the software is constructed to perform the current required function, but with an eye to isolating and localising functionality that can be independently modified, and perhaps reused. Many recent advances in the design of software have not been advances in algorithms, but in the tools and practices of software construction that enable easy modification and reuse of parts.

What relevance does this have to lineage explanations? The dual challenge in software engineering mentioned above comes from the dual role that parts must play in the construction of software, much as the parts in a lineage explanation do. Producing a lineage explanation can be thought of as reverseengineering the modifications that took place in a complex mechanism over time. It is not sufficient to show how some biological phenomenon works (say, for example, how geckos stick to the ceiling), but it must be shown how the mechanism they use is constructed of parts that stay functional as they change. Lineage explanations, by including a continuity dimension, introduce a new set of problems and principles, as they attempt to understand how something works and how it could have changed.

The comparison I am making between certain engineering practices and the kind of reverse-engineering used to explain organismal change over time is not superficial. Similar concepts emerge in both disciplines when trying to discover and understand general principles relating to this dual constraint placed on the parts. Modularity, the focus of much recent discussion in evo-devo (Schlosser and Wagner [2004]; Callebaut and Rasskin-Gutman [2005]), is a key concept in software development practice. Kirschner and Gerhart's list of 'evolvability properties' includes weak linkage, a concept that has many similarities with the idea of loose-coupling and indirection in software development (Kirschner and Gerhart [1998], [2005]). These concepts are connected to the dual role that parts must play in both disciplines: both producing some output and serving as the unit of change. Knowing what kind of structures enable working mechanisms to robustly and easily change over time is relevant to both reconstructing organismal change and designing systems subject to frequent modification.

The analogy can be summarised in the following way. Good software design requires that the software must not only perform a function, but also be easily modifiable. These dual constraints make a big difference to how the software will be constructed. Likewise in biology, understanding both how a mechanism can produce some phenomena, and how that same mechanism was modified over time, changes the kind of explanation given, for both issues must

10 Jacob famously likened the process of evolution to 'tinkering' rather than 'engineering' (Jacob [1977]). Jacob's reasons for classifying something as tinkering rather than engineering suggest that a much of software engineering may rightly be called software tinkering. 
be simultaneously addressed in a lineage explanation. In each case, the additional requirements, over and above explaining or designing how something works, correspond to adding what I have called a continuity dimension. This new dimension gives the components of the mechanisms an additional role as elements of change over time.

Explaining what kinds of structural features enable change is a project of interest to both evo-devo and software development. Note that this is a different question from understanding what kinds of evolutionary processes could have evolved such structures. How modularity might enable future change, and how modularity evolved, are related but distinct issues. The analogy that I made between these two types of pursuits is only possible because both ignore the processes that cause change along the continuity dimension. It doesn't matter why the software was required to be modified; what mattered was that it was modifiable. Likewise, in a lineage explanation, the fact that natural selection caused the change is not part of the explanation. What matters is that we describe the mechanism in a way that we can see how a small modification to some component could have, by whatever process, enabled such a change in the mechanism. I shall return to this issue in more detail in Section 7.

\section{Constraining the Explanations}

The production and continuity requirements constitute the core of a lineage explanation. Without these, there is no lineage explanation. There are, however, a number of important ways that these explanations may be constrained by bringing additional information to bear on the explanation. Doing this is much like what I did to make the puzzle variants in Section 2. Introducing extra constraints on the puzzles made them harder, as there were fewer trajectories that met the imposed conditions. In the case of explanation, introducing constraints likewise reduces the number of possible trajectories. The more we can constrain the possible trajectories, the more plausible our solution will look. Since the structure of a lineage explanation has two dimensions, additional constraints can be introduced in two ways: we can constrain what counts as a valid stage in our explanation, and we can constrain the way that we connect the stages.

The requirement at each stage in a lineage explanation is to show how some particular mechanism performs some task. As I've said, each of these stages is itself an independent explanation about how some mechanism worked. Much of the time these reconstructions will be about mechanisms which no longer exist, so there will be familiar problems of inferring function from structure, or from similarity to features of extant organisms. For example, both lineage explanations I have given invoke analogues to extant organisms. These analogous organisms need not necessarily be part of the particular lineage 
that is being reconstructed. Invoking their existence can serve to increase the plausibility that a mechanism such as this is realistic and workable, without it necessarily being the case that the extant organisms' particular mechanism is homologous with the one being explained. So we can constrain the plausibility of individual stages with respect to whether certain types of mechanisms are, in fact, biologically realisable.

If we work along the other dimension of the explanation, then we can constrain the relation between the different stages of the explanation. This can be done because we may have evidence for, or be able to assume, certain things about the order in which particular stages occurred.

Both examples - the evolution of the eye and the evolution of the feather-imposed some sort of temporal ordering on the stages (in the same way that sort order was imposed on the word puzzle). It wasn't simply that some stages were adjacent to one another; the changes were presumed to go in only one direction. This directionality was imposed in a very different way in each case. Consider the contrasting goals given by the authors of the two examples of lineage explanations:

The first and most crucial task is to work out an evolutionary sequence which would be continuously driven by selection (Nilsson and Pelger [1994]).

What is required is a theory of the origin of feathers that is based on available evidence and that is independent of hypotheses about their presumed ancestral function (Prum [1999]).

This difference in approach is partly explained by the subject matter. Note that the evolution of the eye (at least in this model) is simple in one important way. The adaptive function of the eye remains the same throughout its evolutionary history. At every succeeding stage, the eye more accurately captures the incoming pattern of variation in light.

But, unlike the eye, current adaptive function may differ from original adaptive function. This is exactly the case for feathers. One function that feathers serve in birds today is flight. We can be fairly certain that the precursors of the feather could not have served this same function (Prum [1999]). Feathers are presumed to have performed various other adaptive roles, such as thermoregulation, waterproofing or mate attraction. Adaptive accounts of the evolution of feathers assume that there has been a shift of function over time. So the kind of relationship we want between the stages in our lineage explanation is not necessarily one where the focal trait performs the same function.

This multifunctional history of feathers, in contrast with the clear singular function of the eye, makes the task of lineage reconstruction from an adaptive stance much more difficult. It is not the case that we can clearly track and 
measure one function of feathers over time, as Nilsson and Pelger do with the eye.

Prum's insight was to see that adaptive concerns are not the only way to constrain the directionality of stages in a lineage explanation. He uses facts about the hierarchical nature of feather follicle development to constrain the order of the stages. Certain features must be in place before others are possible. For example, the filaments on the barbs are used to fuse to the rachis during helical growth. So it is presumed that production of this filamentous stage (iii) was prior to the introduction of helical growth in (iv).

So although an optimising assumption-drawn from assumptions about the process of selection - may be used to order the stages, there are other general principles that can be used to order the stages in a lineage explanation. Both Simon's argument for stability of hierarchical construction, and Wimsatt's 'generative entrenchment' provide principled methods of constraining the order of evolutionary change within mechanisms (Simon [1962]; Wimsatt [2001]).

Although lineage explanations such as Nilsson and Pelger's make some optimality assumptions based on the way that natural selection works, it is important to note that the explanations themselves do not include any explicit population-level processes. It is simply assumed that the performance of the task must get better with each successive mechanism, and the stages are ordered as such. Such a simple optimising assumption about the process of natural selection may not hold in many cases. For example, if there is some kind of frequency-dependent selection occurring, these assumptions would be suspect. Nor is it necessary that natural selection be responsible for the optimising process. Lineage explanations for change could well be sought for the kind of changes induced by artificial selection. ${ }^{11}$ As Prum's explanation shows, an uphill adaptive drive is not the only way we apply ordering to the stages. In both cases, the ordering constraint on the stages is supplied by information about the kind of process that is responsible for the evolution of these changes.

There may be other details which can support the claims about certain stages, or the relation between them, such as fossil or phylogenetic resources (Telford and Budd [2003]; Serb and Oakley [2005]). For example, Prum's ordering of the stages in feather evolution derives primarily from assumptions about the hierarchical nature of development (as discussed above). But if good fossil evidence could be produced that showed a particular ordering of forms, this could be used to corroborate, falsify or constrain these assumptions.

Is directionality a necessarily feature of these explanations? No. Sometimes a great deal of insight can be gained by simply knowing that there is some plausible path between certain kinds of mechanisms. Ijspeert et al. use a complex

11 A very simple example of this would be the reconstructions of lineages provided in Dawkins' 'Biomorph' evolution, which is explicitly an agent-driven optimising process (Dawkins [1986]). 
salamander-like robotic model to demonstrate the changes required in a neural circuit for it to switch from generating the limb movements for swimming to those for walking (Ijspeert et al. [2007]). They argue that this tells us something about the switch from aquatic to terrestrial locomotion. There is neither an adaptive nor a developmental constraint on their model. What is shown is that a complex model of the neural pattern generation (based on empirical work) can be modified to switch between these two modes of locomotion. Directionality of some kind can provide extra information to constrain the relationship between stages, but the central requirement is still one of continuity.

\section{Operational and Generative Lineages}

Thus far, I have claimed that the explanation for adaptive complexity (the eye), and the explanation for the origin of an evolutionary novelty (the feather) have a great deal in common. Both of these explanations possess a core structural similarity that identifies them as lineage explanations. But there are obviously some differences between the explanation for feather evolution and that for eye evolution. The previous section explained some of these differences by appealing to different ways that the trajectories could be constrained. In this section, I look at a deeper difference between the two. The relation between the focal phenotypic trait and the mechanism is very different depending on whether one is interested in adaptation or development. Understanding this difference sheds some light on why developmental details have a special role in the explication of evolutionary novelties.

Consider the difference between these two questions:

(1) How does that car work?

(2) How was that car manufactured?

Both questions could be answered by some sort of mechanistic explanation. An explanation of how the car works would include some description of the parts of the car, how they are organised, how they operate, and as a result how the car moves, steers and stops. An explanation of how the car was manufactured would also include a description ofthe parts, operations and their organisation. But this description of components would not include (for the most part) the components that one finds in a car. Rather, they would be the kinds of parts and processes that are found in a manufacturing plant. So, although both questions are about cars, and both explanations are about mechanisms, the role that the car plays in each explanation is different. In the first case, the components of the car are the components of the mechanism and the phenomena that these components produce is the behaviour of the car. In the second case, the components of the car-its parts, processes and organisation-are produced 
by the mechanism, and this mechanism is, for the most part, something quite different from the car.

Note that, given either of these mechanisms, we can still make sense of a lineage explanation in which they might play a role. We can ask how changes in the car components affected the operation over time, and we can ask how changes in the components of the manufacturing plant affected the generation of cars over time. In both cases, we might say that such a coming up with such a series of changes describes 'evolution' of cars. But these would be quite different-looking explanations.

The difference picked out by these two explanations is apparent when we contrast the eye and feather examples. If we focus on a single stage in the two examples of lineage explanations, it becomes apparent that the eye plays a different role in the lineage explanation than the feather does. The eye is the mechanism, and the phenomena that it produces are due to the operation of the eye mechanism. The feather, however, is what is generated by the feather follicle - the feather is what the follicle mechanism produces. The focal trait (the eye or the feather) plays a different role in each case. It is either the mechanism that is operating, or what is generated by the mechanism.

This different role that the focal phenomenon plays is important when we examine how phenotypic novelties are explained. Explaining the origin of novelties poses a problem, because a requirement of these explanations is a degree of similarity between stages - what I've called the continuity requirement. The problem is addressed by switching the role that the focal trait plays in the lineage explanation. Rather than giving a continuous series of mechanisms that show how a trait's operation changed over time, instead we give a continuous series of mechanisms showing changes in how that trait was generated over time.

Why should a shift from the focal trait as something operating to something being generated help explain novelties? Recall the case of the car. A description of the mechanisms that manufacture a car will, for the most part, consist of very different components than those existing in the mechanism that explains the operation of the car. So, if our concern was that there was some discontinuity in the how the car operated, a lineage of manufacturing mechanisms is not constrained to telling this story in terms of parts that appear in the car. The upshot is that it is possible to satisfy a continuity constraint by the relations between the components of a generative decomposition, even when there is no continuity between the components in an operational decomposition.

We can see how this works by comparing the two examples. In the first example, the mechanism in question is the operation of the eye. In order to provide continuity between the stages, Nilsson and Pelger decompose the eye into certain components. This decomposition results in components that are part of the structure and function of an eye: a lens, a retina, etc. This is very different from the second example. Here, the mechanism in question is the 
generation of the feather-something produced by the operation of the feather follicle. So our decomposition is into the components at the level of the feather follicle rather than the feather itself. We are still able to explain the changes in the feather because the feather follicle generates the feather. But note the difference: we can now meet the continuity requirement by describing changes in the follicle, rather than the feather itself. The generative decomposition into a feather follicle provides a level of description that has a continuity not available at the focal level of the feather.

Shifting to a generative decomposition makes our focal trait the form or morphology of some part of the organism. We don't try and describe continuity at this level, for this is the phenomenon that the generative mechanism produces. Continuity is instead provided by showing changes in the components making up the mechanisms that produced the form. As I said above, the parts we use to understand the manufacturing of something may be entirely different from those that the manufacturing process produces. So any large, discontinuous changes in the phenomenon produced by the mechanism (novelties, for example) are not a concern. Developmental details are essential for these explanations of novelty, for it is only by using developmental information that we can give these generative decompositions.

\section{Explaining Change Without Populations}

In this section, I take a closer look at how lineage explanations relate to other kinds of explanations. I argue that they are genuine explanations of evolutionary change, but that they do not include populations. To see why this is so, it is important to distinguish two very different questions we might ask about evolutionary change. The first question concerns the processes driving change in populations over time. This will include such things as migration, mutation and natural selection. The second question is one about individuals rather than populations, and is the focus of this paper. This question ignores what processes drove the change and instead pays attention to how the components that produced some phenotypic phenomenon (morphology, behaviour, etc.) could be gradually modified to reflect some series of changes in that phenomenon (a fossil record, or perhaps some putative adaptive sequence). Both of these questions are about evolutionary change. But they are quite different, non-competing explanatory patterns.

Recognising the population as the unit of explanation was central to understanding natural selection (Lewontin [1983]). Before Darwin, explanations for population change - such as that given by Lamarck - were simply a summation of changes within individuals. Although it is change at the population level that must be invoked when talking of natural selection, population-level change is not the only kind of change of interest in biology. The kind of explanations 
under focus in this paper are those that tell us the changes that could produce differences between individuals, not populations. Explaining what underwrites the differences between individuals is a common pursuit in biology. ${ }^{12}$

Consider how we might explain an illness such as cystic fibrosis. First we pick some symptomatic phenomenon that differs between individuals that do and don't have cystic fibrosis. We might identify differences at many different levels of organisation; they may exist in the function of organs, cells or proteins. We then explain these differences by showing how a change in the operation of some underlying mechanism produced these differing phenomena. For example, we might explain the difference in cellular function between those with cystic fibrosis and those without by showing how a change in protein shape affects the transport of chloride across the cell membrane. Or we might explain the build-up of mucus in the lungs and subsequent bacterial infection by reference to this same lower level phenomenon we have just explained - a change in the ability to transport chloride. These explanations tell us the changes in a mechanism that produce the difference between healthy individuals and those with cystic fibrosis.

This explanation invokes no population processes. It demonstrates how some small modifications to a mechanism operating in an individual can produce differences in some focal phenomenon. It is an individual-level explanation, for what is being described are mechanisms operating within individuals, how they function and how they can be changed. The processes of migration, drift and natural selection play no role here. We might want to give an explanation of why the recessive allele underlying cystic fibrosis continues to remain in a population, but this is to engage in a very different kind of explanation.

This example demonstrates an important (and perhaps obvious) point. Explaining how, in general, some phenomenon is produced by a biological mechanism provides us with capability to explain what underlies individual differences in that phenomenon. We began with an explanation of how some cellular mechanism worked, and in virtue of some changes to the mechanism, we explained how a different sort of phenomenon (the cystic fibrosis) could be produced. This is unsurprising as understanding how something works is exactly the information we need to know in order to understand how it can be manipulated or changed.

This connection seems well founded, as it is widely accepted that underlying these mechanistic explanations is a difference-making, or manipulationist,

12 It is important to note that the difference between population-level explanations and lineage explanations discussed here is not the same as the contrast drawn between explanations for evolutionary change by both Lewontin ([1983]) and Sober ([1984]). In both Lewontin and Sober's case, the explanations for change are competing, as they have the same explananda. Lineage explanations, by contrast, seek to explain something different than population change. So they are not simply Lewontin's 'transformational' explanations or Sober's 'developmental' explanations. (Thanks to an anonymous reviewer for pointing this out.) 
account of explanation (Glennan [2002], [2005]; Woodward [2002]; Craver [2007]). On this account, to explain some target phenomenon is to show what it depends on and the structure of this dependence. This structure consists of a set of generalisations showing how the phenomenon changes under various interventions or manipulations. This structure of dependence gives us the resources for answering what-if-things-had-been-different questions. So the parts, processes and organisation of the mechanism explain how it produces a particular phenomenon because changes to those same parts, processes and organisation would result in a change in the phenomenon produced. Equally, understanding how a particular phenomenon is produced by some mechanism provides us with a set of components whose modification can explain why different individuals vary in the production of this phenomenon. This is just what we saw in the cystic fibrosis case: understanding the mechanism underlying a symptom of the disease allowed us to explain what underwrote the differences between individuals with the disease and those without it.

Note what this observation entails for lineage explanations. The components of the mechanism play a dual role, because in lineage explanations the use of difference-making occurs in both dimensions.

First take the production dimension. Any explanation of how some mechanism works (of how it produces some phenomenon) is going to contain a description of certain parts and processes. The description is explanatory because it shows how this phenomenon depends on the parts, processes and organisation of its constituent components. These dependencies are reflected by how changes in these various components produce differences. This is just standard mechanistic explanation, and this relates to the production dimension of a lineage explanation - it tells us how that particular mechanism works.

But difference-making also plays a role in the continuity dimension of these explanations as well. For we explain how the mechanism could have changed over time by showing how small changes in the parts, processes and organisation occurred over time. Rather than identifying differences between individuals within a population (as was the case with the cystic fibrosis explanation), lineage explanations lay out of a series of such models that are related by descent, and rather than identifying the small changes in mechanisms responsible for differences between a healthy and a diseased individual, lineage explanations show how small changes between ancestral and derived mechanisms could have produced different behaviour, physiology and morphology.

Although both dimensions engage in difference-making, there is an important difference between the two. As I have said, the production dimension is simply a mechanistic explanation. It shows how some putative mechanism could have functioned, or actually does function. The use of difference-making in the continuity dimension is different. For although the difference between stages is contrastive-showing how a change in some component produced a 
difference in phenomena-here we are asserting that the change was actually made, rather than it being some in-principle intervention. This change is what took the mechanism from one stage to the next. Furthermore, although we are assuming that a change like this took place, we explicitly do not say what caused these changes. Whatever processes actually induced the change do not play a direct part in the explanation. The same lineage explanation for some biological change could be given, whether the process driving that change was natural selection, artificial selection or genetic engineering. ${ }^{13}$ So although the production dimension is explicitly causal, the continuity dimension to a lineage explanation provides no causal connection between the processes that made the change between the mechanisms.

It is important that a lineage explanation of evolutionary change is not in conflict with a population-level explanation that invokes natural selection. Both explain change over time, but they are asking very different questions (rather than answering the same question in different ways). One explains how populations change over time. The other explains how complex mechanisms can be modified gradually, yet continuously produces some specified outcome, be it behavioural, physiological or morphological. The lack of an explicit causal connection between adjacent stages along the continuity dimension is exactly the 'causal gap' that an explanation by natural selection can fill.

The key assumption that connects these individual-based explanations with population processes is that the mechanistic descriptions in each stage are representative of some population or set of individuals, as they were in the cystic fibrosis example. A lineage explanation assumes that each such mechanistic description in the lineage was representative of the population, and enabled the next variation in the lineage to arise. In practice, it may be that the prototypes used to reconstruct a lineage are, in fact, particulars - such as fossils or a model organism. If we wish to generalise our results then, again, we have to assume that they are, or were, representative of some population. So a lineage explanation reconstructs a chain of individual (descriptions of) mechanisms, where these mechanisms are representative of those possessed by organisms assumed to have been fixed in the population.

\section{Conclusion}

Adaptationist: Individuals don't evolve. Populations do. Species are the effects of the evolution of populations

Structuralist: Individuals don't evolve. Ontogenies do. Characters are the effects of the evolution of ontogenies (Amundson [2005]).

13 Lineage explanations could likewise be given for non-biological changes, such as the historical series of improvements to some engineered invention. 
Amundsen's characterisation of the structuralist and adaptationist positions captures the differing explanatory interests one might have about change over evolutionary time. Lineage explanations capture this second explanatory interest. But I have argued that this second explanatory interest also comes in an adaptive flavour-lineage explanations where the mechanisms in question produce some adaptive behaviour, and the directionality of the stages are constrained by optimising assumptions about natural selection.

In either case, no population processes are present, but the explanations still answer important questions about evolutionary change. Showing how complex functioning mechanisms can gradually change really does present a puzzle, and is something genuinely in need of explanation. Knowing how a complex assemblage of parts does some particular task is one thing. Knowing how these parts and their organisation could be modified to improve or change the performance of that task is a different challenge.

There is more than one kind of explanation for change over time, because there is more than one kind of question that we can ask. One is about the population-level processes that are responsible for that change. The other is about the properties of the individuals undergoing change: how can they both change gradually and continue to function? It is a mistake to think that all relevant questions about evolutionary change can be subsumed under a single explanatory pattern.

\title{
Acknowledgements
}

Many thanks to Kim Sterelny, Peter Godfrey-Smith, John Matthewson, Patrick Forber, Lindell Bromham and Rob Lanfear for advice, comments and suggestions. Two anonymous referees also helped make this a much better, and more comprehensible paper.

\author{
Philosophy Program \\ Research School of Social Sciences; \\ Centre for Macroevolution and Macroecology \\ School of Botany and Zoology \\ Australian National University \\ Canberra, ACT 0200 \\ Australia
}

\section{References}

Amundson, R. [2001]: 'Adaptation and Development', in S. H. Orzack and E. Sober (eds), Adaptationism and Optimality, Cambridge: Cambridge University Press.

Amundson, R. [2005]: The Changing Role of the Embryo in Evolutionary Thought: Roots of Evo-Devo, New York, NY: Cambridge University Press. 
Autumn, K. and Peattie, A. M. [2002]: 'Mechanisms of Adhesion in Geckos', Integrative and Comparative Biology, 42, pp. 1081-90.

Bechtel, W. and Abrahamsen, A. [2005]: 'Explanation: A Mechanist Alternative', Studies in History and Philosophy of Science Part C: Studies in History and Philosophy of Biological and Biomedical Sciences, 36, pp. 421-41.

Bechtel, W. and Richardson, R. C. [1993]: Discovering Complexity: Decomposition and Localization as Strategies in Scientific Research, Princeton, NJ: Princeton University Press.

Callebaut, W. and Rasskin-Gutman, D. (eds.). (2005). Modularity: Understanding the Development and Evolution of Natural Complex Systems, Cambridge, MA: MIT Press.

Craver, C. F. [2007]: Explaining the Brain, Oxford: Clarendon Press.

Darden, L. [2002]: 'Strategies for Discovering Mechanisms: Schema Instantiation, Modular Subassembly, Forward/Backward Chaining', Philosophy of Science, 69, pp. S354-S365.

Darden, L. [2005]: 'Relations among Fields: Mendelian, Cytological and Molecular Mechanisms', Studies in History and Philosophy of Science Part C: Studies in History and Philosophy of Biological and Biomedical Sciences, 36, pp. 349-71.

Dawkins, R. [1986]: The Blind Watchmaker, Harlow: Longman Scientific \& Technical.

Glennan, S. S. [1996]: 'Mechanisms and the Nature of Causation', Erkenntnis, 44, pp. 4971.

Glennan, S. S. [2002]: 'Rethinking Mechanistic Explanation', Philosophy of Science, 69, pp. S342-S353.

Glennan, S. S. [2005]: 'Modeling Mechanisms', Studies in History and Philosophy of Science Part C: Studies in History and Philosophy of Biological and Biomedical Sciences, 36, pp. 443-64.

Ijspeert, A. J., Crespi, A., Ryczko, D. and Cabelguen, J. M. [2007]: 'From Swimming to Walking with a Salamander Robot Driven by a Spinal Cord Model', Science, 315, pp. 1416-20.

Jacob, F. [1977]: 'Evolution and Tinkering', Science, 196, pp. 1161-6.

Kirschner, M. and Gerhart, J. [1998]: 'Evolvability', Proceedings of the National Academy of Sciences, 95, pp. 8420-7.

Kirschner, M. and Gerhart, J. [2005]: The Plausibility of Life: Resolving Darwin's Dilemma, New Haven, CT: Yale University Press.

Lewontin, R. [1983]: 'Darwin's Revolution', New York Review of Books, 30, pp. 2172.

Machamer, P., Darden, L. and Craver, C. [2000]: 'Thinking About Mechanisms', Philosophy of Science, 67, pp. 1-25.

Müller, G. B. and Wagner, G. P. [2003]: 'Innovation', in B. K. Hall and W. M. Olson (eds), Keywords and Concepts in Evolutionary Developmental Biology, Cambridge, MA: Harvard University Press.

Nilsson, D. E. and Pelger, S. [1994]: 'A Pessimistic Estimate of the Time Required for an Eye to Evolve', Proceedings of the Royal Society of London Series B-Biological Sciences, 256, pp. 53-8. 
Peel, A. and Akam, M. [2003]: 'Evolution of Segmentation: Rolling Back the Clock', Current Biology, 13, pp. R708-10.

Prum, R. O. [1999]: 'Development and Evolutionary Origin of Feathers', Journal of Experimental Zoology, 285, pp. 291-306.

Raff, R. A. [1996]: The Shape of Life: Genes, Development, and the Evolution of Animal Form, Chicago, IL: University of Chicago Press.

Rothschild, M., Schlein, J., Parker, K., Neville, C. and Sternberg, S. [1975]: 'Jumping Mechanism of Xenopsylla-Cheopis. 3. Execution of Jump and Activity', Philosophical Transactions of the Royal Society of London Series B, 271, pp. 499-515.

Schlosser, G. and Wagner, G. P. [2004]: Modularity in Development and Evolution, Chicago, IL: University of Chicago Press.

Serb, J. M. and Oakley, T. H. [2005]: 'Hierarchical Phylogenetics as a Quantitative Analytical Framework for Evolutionary Developmental Biology', Bioessays, 27, pp. 115866.

Simon, H. A. [1962]: 'The Architecture of Complexity', Proceedings of the American Philosophical Society, 106, pp. 467-82.

Sober, E. [1984]: The Nature of Selection: Evolutionary Theory in Philosophical Focus, Cambridge, MA: Mit Press.

Suzuki, Y. and Nijhout, H. F. [2006]: 'Evolution of a Polyphenism by Genetic Accommodation', Science, 311, pp. 650-2.

Tabery, J. G. [2004]: 'Synthesizing Activities and Interactions in the Concept of a Mechanism', Philosophy of Science, 71, pp. 1-15.

Telford, M. J. and Budd, G. E. [2003]: 'The Place of Phylogeny and Cladistics in EvoDevo Research', International Journal of Developmental Biology, 47, pp. 479-90.

Thompson, D. A. W. [1961]: On Growth and Form Abridged edition, edited by J. T. Bonner. Cambridge, UK: Cambridge University Press.

Truman, J. W. and Riddiford, L. M. [1999]: 'The Origins of Insect Metamorphosis', Nature, 401, pp. 447-52.

Wagner, G. P. [2000]: 'What is the Promise of Developmental Evolution? Part I: Why is Developmental Biology Necessary to Explain Evolutionary Innovations?', Journal of Experimental Zoology, 288, pp. 95-8.

Wehner, R. [2003]: 'Desert Ant Navigation: How Miniature Brains Solve Complex Tasks', Journal of Comparative Physiology A, 189, pp. 579-88.

Wimsatt, W. C. [2001]: 'Generative Entrenchment and the Developmental Systems Approach to Evolutionary Processes', in S. Oyama, P. E. Griffiths and R. D. Gray (eds), Cycles of Contingency, Cambridge, MA: MIT Press. pp. 219-38.

Winther, R. G. [2005]: 'Evolutionary Developmental Biology Meets Levels of Selection: Modular Integration or Competition, or Both?', in W. Callebaut and D. Rasskin-Gutman (eds), Modularity: Understanding the Development and Evolution of Natural Complex Systems, Cambridge, MA: MIT Press. pp. 61-97.

Woodward, J. [2002]: 'What Is a Mechanism? A Counterfactual Account', Philosophy of Science, 69, pp. S366-S377.

Yu, M., Wu, P., Widelitz, R. B. and Chuong, C.-M. [2002]: 'The Morphogenesis of Feathers', Nature, 420, pp. 308-11. 